\title{
Transforming a Monolithic Sheet of Nitinol into a Passive Reconfigurable Tactile Pixel Array Display at Braille Resolution
}

\author{
Tigmanshu Bhatnagar $^{1}$, Nicolai Marquardt ${ }^{1}$, Mark Miodownik ${ }^{2}$ and Catherine Holloway ${ }^{1}$
}

\begin{abstract}
Shape changing materials create a unique opportunity to design reconfigurable tactile display actuators. In this paper, we present a method that transforms a single thin monolithic sheet of Nitinol into a passive reconfigurable tactile pixel array at Braille resolution. We have designed a $27 \times 27$ tactile pixel array in which each pixel can be selectively actuated with an external source of heat. The pixels rise $0.4 \mathrm{~mm}$ vertically with a peak blocked force of $0.28 \mathrm{~kg}$ and have an average blocked force of $0.23 \mathrm{~kg}$ at room temperature. After cooling, the pixels can be mechanically reconfigured back to their flat state for repeatable actuation. We demonstrate this actuator's interactive capabilities through a novel erasable tactile drawing interface.
\end{abstract}

\section{INTRODUCTION}

Shape changing smart materials are able to reversible change their shape or dimensions in response to an external stimulus such as heat, light, pressure or electricity. These materials can intrinsically act as actuators that are useful in compact mechanical systems. A tactile display is such a compact mechanical haptic interface that creates changes in tactile parameters such as surface texture, roughness and temperature to communicate information [1]. Nitinol (alloy of Nickel and Titanium in nearly equal ratio) which is a shape memory alloy (SMA) has distinct advantages that are well suited for tactile displays. Shape memory effect in Nitinol occurs due its ability to reconfigure its crystal structure through a temperature induced transformation from its malleable martensite to its memorized and stiffer austenite state. The resulting motion from the shape memory effect is completely silent and low voltages of 3-12V are sufficient for this actuation. Nitinol also has high recoverable strains (up to $10 \%$ ) and high stress resistance (up to 700MPa) [2] which are favorable to achieve the force and motion required for the actuators of tactile displays, within their size and space constraints. This high-power density can continue over a long thermo-mechanical fatigue life making Nitinol suitable for long term practical use [2]. The main disadvantage of using SMAs is that it requires to be heated for shape-change, which takes time and energy causing a low actuation speed and refresh rate. However, these limitations can be addressed by the mechanical and thermal design of the actuator.

*This work is funded by the GRS Scholarship from UCL and supported by the UKAID funded AT2030 program which is led by the Global Disability Innovation Hub.

1Tigmanshu Bhatnagar, Nicolai Marquardt and Catherine Holloway are with UCL Interaction Centre, 66-72 Gower St., Bloomsbury, London, WC1E6EA, UK. Emails: t.bhatnagar.18@ucl.ac.uk, n.marquardt@ucl.ac.uk, c.holloway@ucl.ac.uk

${ }^{2}$ Mark Miodownik is with UCL Mechanical Engineering, Roberts Building, Torrington Place, London, WC1E7JE, UK. Email: m.miodownik@ucl.ac.uk

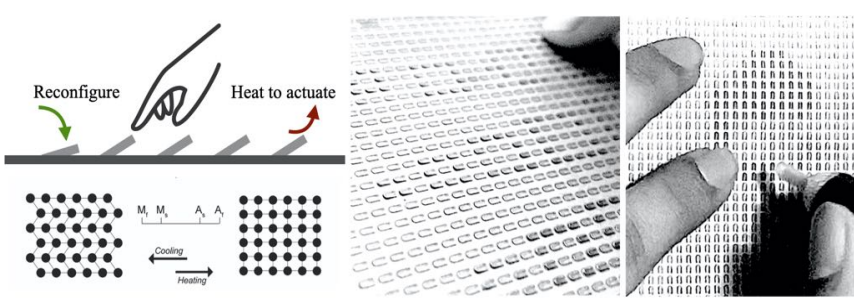

Figure 1. Tacilia is a passive shape chaning display made from a single sheet of Nitinol. It leverages the shape memory effect of the material. One can manually read, draw and erase Braille and tactile diagrams on Tacilia.

Nitinol has been previously explored as a mechanical actuating element for tactile displays in multiple forms and configurations. A common approach has been to use Nitinol wires that are actuated through resistive heating. Actuator configurations vary from wires that bend cantilever beams to raise and lower pins [3], to Nitinol springs that compress to create reciprocating motion for the same effect [4]. Fewer design configurations have explored Nitinol in its sheet form for tactile actuators. For instance, $\mathrm{Xu}$ et al. [5] developed a tactile display with meandering Nitinol films glued to a bias spring on one side and pins on the other. In their system heat from an external heater array, changed the shape of the Nitinol film which caused the pin to move on one side. The bias spring reconfigured the system back, creating a vibrotactile array of pins. Hafez et al. [6] proposed the concept of using a monolithic sheet of SMA with integrated actuators which are in the form of cantilever beams that work in bending. However, this concept has not been implemented.

There are 4 requirements to design Nitinol based mechanical actuators. First, Nitinol is available and can be formed in a variety of shapes (sheet, wires etc.), and therefore creative exploration for a suitable form and configuration is required. Second, actuation through the shape memory effect is a trained behavior of the material that occurs after a heat treatment process. The training process has to be adapted to the actuator's design. Third, heat is required to activate the shape memory effect. Therefore, actuation rate depends on the rate at which the material is heated. Heating of Nitinol can be done directly by passing current in the material through resistive heating or indirectly through conduction, convection, or radiation from an external source of heat. Subsequently, refresh rate will depend on how quickly it can be cooled. Cooling can happen naturally in atmosphere, or it can be forced through Peltier elements and fans. Finally, oneway shape memory effect is more repeatable and stable than two-way shape memory effect [7]. Therefore, external mechanical force is required to reconfigure the alloy back to its original form. 
In this paper, we extend the design space of tactile display technologies based on Nitinol. We present a method that transforms a single monolithic sheet of Nitinol into a passive array of tactile pixels at Braille-resolution that can be selectively actuated. Pixels on the sheet are cut by a laser in the form of cantilever beams and are trained to bend out-ofplane when heated, due to the shape memory effect. In the $27 \times 27$ tactile pixel array, each pixel rises an average of $0.4 \mathrm{~mm}$ out-of-plane, which is perceivable. It maintains this position and can resist $0.23 \mathrm{~kg}$ force at room temperature. The pixels can be mechanically reconfigured back to their flat state with a compressive mechanical force for repeatable actuation. To demonstrate the capabilities of this display called Tacilia - we present a novel erasable tactile drawing interface. This interface (Figure 1, right) allows users to haptically draw low resolution erasable tactile diagrams using a hot-air-jet pencil.

\section{Design, Fabrication And Training of TACilia}

In this section, we will discuss the design of Tacilia and present details of its fabrication and training process.

\section{A. Requirements for Tactile Readability}

There are three primary requirements for the tactile readability: the relative position of tactile pixels, their height above the surface and their blocked force, that is the force that the pixels have to resist while they are being felt. Standard dimensional requirements for the position of the tactile pixels (dots) relative to each other for Braille have been previously documented [8]. These specifications have also been used for tactile graphics to convey non-textual information such as maps, graphs and scientific diagrams. Following these will result in a display that is suitable also for blind and partially sighted readers. The vertical and horizontal inter-pixel distance is $2.5 \mathrm{~mm}$. The pixel height is in the range of $0.5 \pm 0.1 \mathrm{~mm}$ and the diameter of the base should be $1.5 \pm 0.25 \mathrm{~mm}$. The blocked force should be in the range of $0.5 \mathrm{~N}-2.5 \mathrm{~N}$ [9].

\section{B. Material Properties of Nitinol}

Shape memory effect in Nitinol occurs due to a temperature induced reversible transformation in its crystal structure [10]. This transformation is marked by four temperature points: Austenite Finish $\left(\mathrm{A}_{\mathrm{f}}\right)$, Austenite Start $\left(A_{s}\right)$, Martensite Start $\left(M_{s}\right)$, and Martensite Finish $\left(M_{f}\right)$. At temperatures below $\mathrm{A}_{\mathrm{s}}$, the martensitic structure (rhombic arrangement) of the alloy can form twins to accommodate external strains without breaking the atomic bonds [10]. Therefore, the material can be mechanically deformed. However, when the temperature of the material rises above $A_{s}$, the austenite form of the alloy begins to be thermodynamically favored and by $A_{f}$ the atoms move to a rigid austenitic structural arrangement (square). Through training, a distinctive shape can be associated with this austenitic structure so that whenever the material is heated above $A_{f}$, it snaps to this memorized shape. Finally, when the material is cooled below the $\mathrm{M}_{\mathrm{f}}$, it begins to regain its martensitic form without changing the austenitic shape. Below $\mathrm{M}_{\mathrm{s}}$ it can be mechanically deformed again.

For our proof of concept we use a sheet of Nitinol from Fuxus ${ }^{\circledR}$ with $51 \% \mathrm{Ni}$, an activation temperature of $40 \pm 5^{\circ} \mathrm{C}$ and dimensions of $100 \times 100 \times 0.3 \mathrm{~mm}$. We conducted ASTM
E8 Tensile Tests with a Zwick Roell Proline tensile testing machine to estimate the Young's modulus and Ultimate Tensile Strength of the material. From an average of three samples, the Modulus of Elasticity was $42.52 \mathrm{GPa}$, its Ultimate Tensile Strength was $965.79 \mathrm{MPa}$, and the Elongation at Break was $7.28 \%$. Other relevant parameters are referred from existing literature [11].

\section{Design of the Tactile Pixel Array}

On a single sheet of Nitinol, we can pixels that bend out of the surface. To achieve the tactile readability requirements, an array of small U-shaped cantilever beams can be cut and trained to bend out of plane. When packed in an array with an inter pixel distance of $2.5 \mathrm{~mm}$, these cantilevers reach a length of $2 \mathrm{~mm}$ with a width of $1 \mathrm{~mm}$, while maintain the overall strength of the sheet. Assuming that the cantilever pixels deform in pure bending, a static nonlinear FEA simulation using Solidworks 2020 was performed to evaluate the strains that will develop when the pixels bend. Using the material properties of the sample and an external load which bends the pixels so that its tip rises by $0.5 \mathrm{~mm}$, maximum equivalent strain of $4.7 \%$ was observed at the base of the cantilevers (Figure 2). This value is much lower than the maximum strain limit of our sample. Therefore, with this design, the pixels can theoretically reach the required height with the shape memory effect. With these dimensions, we are able to pack an array of $27 \times 27$ (729) pixels on the sample $100 \times 100 \mathrm{~mm}$ sheet (Figure 2). Increasing the resolution by reducing the length of the cantilevers to pack them closer to each other will reduce their propensity to bend to the required height. Packing them closer to each other with these dimensions will make it unsuitable for reading Braille. It would also reduce the sheet's strength and may also lead to inaccuracies while actuating the pixels. Hence, further optimization of the resolution was not attempted.

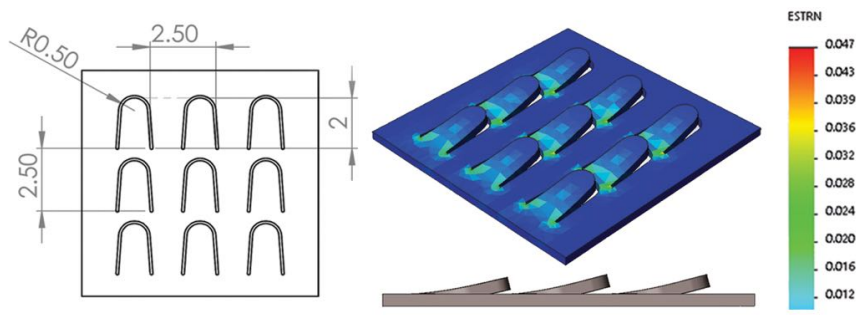

Figure 2. Dimensional drawing and FEA simulation of the equivalent strain of the U-shaped cantilever pixel array when they are bend.

\section{Fabrication at Braille Resolution}

To cut these cantilevers we use a StarFemto StartCut Tube 60W Femtosecond Cold Laser Cutting Machine. The U-shape pixels are oriented in the rolling direction of the sheet to optimize its strength at their base. The parameters to cut the sheet in a single pass were identified through a trialand-error iterative testing on a sample blank. This involved tweaking parameters of the laser so that it was able to cut the sheet with minimal kerf. Optimal results were achieved with the focus of the laser head adjusted to a beam diameter of $80 \mu \mathrm{m}$. At a frequency of $995 \mathrm{kHz}$ with a single burst and estimated output power of $45 \mathrm{~W}$, the sheet was cut in a single pass (Figure 3). 


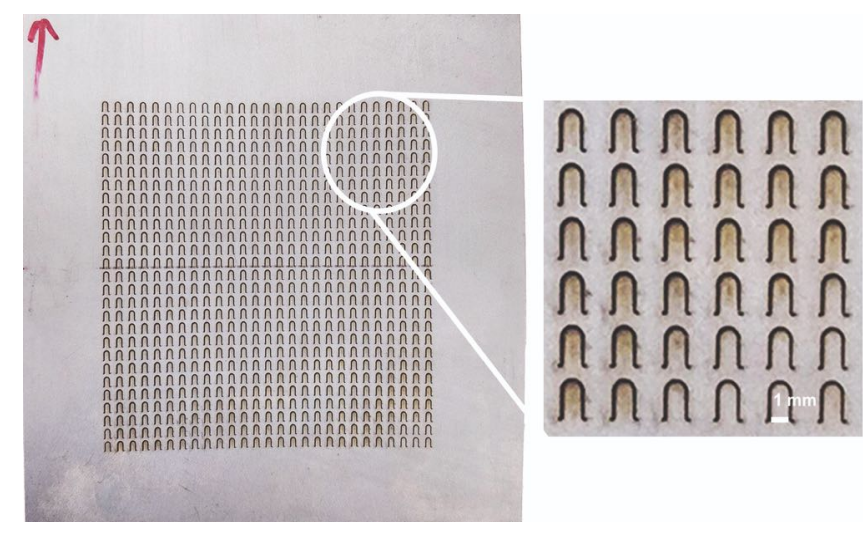

Figure 3. Laser cut sheet with 729 (27x27) cantilever beams (tactile pixels) at Braille resolution.

\section{E. Training the Pixels to Achieve Actuation and Mechanical Reconfigurability}

We adapted previously prescribed instructions for the process of one-way shape memory training to train the pixels [12]. The first step of the process was to bend and constrain the cantilever pixels to their actuated state below the $A_{s}$. This was achieved by pushing the cantilevers to their actuated state and then constraining them by introducing a thin titanium wire of $0.25 \mathrm{~mm}$ between the pixels and the sheet at room temperature. In this configuration, the pixels were bend so that their tips are $0.55 \mathrm{~mm}$ above the surface. The sheet was then clamped between two steel plates to prevent the wires from slipping out. The assembly was kept in a furnace at $500^{\circ} \mathrm{C}$ for 30 minutes after which it was quenched in a water bath. With this process, the sheet was retrained to remember the new austenite state or the bend state of the pixels.

However, with this process the pixels were observed to be stiff at room temperature. They did not reconfigure to their flat state and snapped back to their actuated state when the compressive force was released. In order to increase the malleability of the material, the pixels were again constrained in their actuated state and clamped between two plates. The assembly is kept in the furnace at $500^{\circ} \mathrm{C}$ for 48 hours following instructions to create precipitates in Nitinol [13]. It was then taken out and allowed to cooled down naturally to room temperature. The pixels retained their actuated state however, the annealing process made the pixels deformable at room temperature (Figure 4 (right)). Furthermore, due to the mechanical constrain during the heat treatment process, austenitic memory of the material was not lost. Therefore, when the pixels are heated above the $A_{s}$, they bend out of plane again and maintain the bend position (Figure 4 (left)).

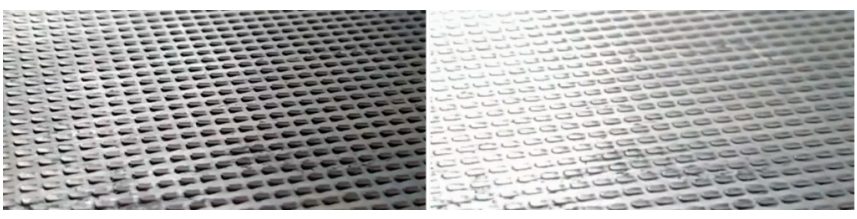

Figure 4. Actuated (left) and flattened (right) tactile pixels.

\section{Performance of the Tactile Pixels}

We experimentally evaluated the performance of the pixels by measuring their peak displacement, vertical blocked force and the force required to reconfigure with respect to the material's temperature.

\section{A. Apparatus}

To heat Tacilia, we used a heater bed from a 3D printer. The heater was attached to sheet with a double sided Kapton tape. Power was supplied to the heater with a bench top power supply and the temperature of the sheet was monitored by a $10 \mathrm{k}$ ohm thermistor attached near the pixel that was being tested. To measure the vertical actuation force, the target pixel was kept under a $20 \mathrm{~kg}$ load cell that had a nonconductive probe. Load cell data was calibrated and gathered using a HX711 amplifier and an Arduino Uno on a MacBook Pro 2015 computer. To measure the vertical displacement of the pixels, we used a Mitutoyo ${ }^{\circledR}$ Dial Gauge. The room temperature for the tests was $26^{\circ} \mathrm{C}$.

\section{B. Procedure}

Current from the power supply to the heater $(12 \mathrm{~V}, 3 \mathrm{~A})$, increased the temperature of the sheet and actuated all the pixels at once. Force generated by a single randomly chosen pixel and temperature of the SMA sheet was measured until the displacement and blocked force of the pixels saturated. The peak-displacement, rise in temperature of the pixels and force variations are recorded after which power was cut off and the sheet was removed from the heater to naturally cool to room temperature. This procedure is repeated for 5 random pixels.

\section{Results}

The vertical force from the pixel varied significantly with the rise in temperature. From room temperature and a completely flat state, until the rated activation temperature of $40^{\circ} \mathrm{C}$, the average lift force increased from $0 \mathrm{~kg}$ to $0.23 \mathrm{~kg}$ and the peak vertical displacement of the pixels was $0.4 \mathrm{~mm}$. No noticeable vertical displacement is observed beyond this value. However, the blocked force further increased and began to saturate above this temperature (Figure 5) so that the average peak lift force generated by the pixels was $0.28 \mathrm{~kg}$, at around $49^{\circ} \mathrm{C}$.

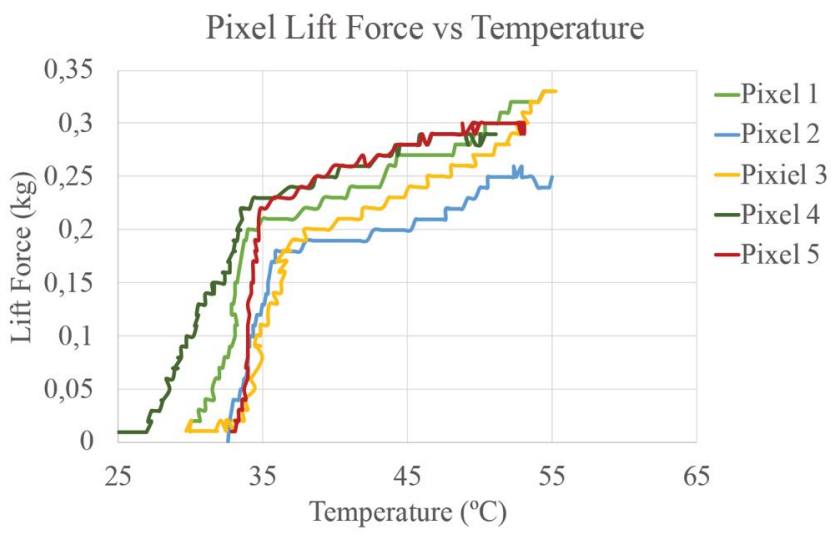

Figure 5. Plots of force vs temprature from 5 randomly selected pixels shows how the lift force from each pixel varies with temperature.

When the sheet was allowed to cool naturally, the pixels could be pushed back to their flat state at approximately $38^{\circ} \mathrm{C}$ temperature of the material. Above $38^{\circ} \mathrm{C}$, when the pixels were compressed, they had sprung back close to their 
actuated state. However, if the pixels were not pushed below $38^{\circ} \mathrm{C}$, they maintained their bend position with an average resistive force of $0.23 \mathrm{~kg}$. At $26^{\circ} \mathrm{C}$ room temperature, the sheet naturally cools down at a rate of $3.61^{\circ} \mathrm{C} / \mathrm{s}$. This means that it takes $3 \mathrm{~s}$ for the pixels to cool to a temperature suitable for safe interaction [14] from $49^{\circ} \mathrm{C}$. Table 1 provides an overview of these results. We utilize these features and discuss them in the next section referring specifically to the performance of the sheet as a display when used as an erasable tactile drawing interface.

TABLE I. OVERVIEW OF THE PIXEL's PERformance

\begin{tabular}{|c|c|}
\hline Parameter & Value (Mean / SD) \\
\hline $\begin{array}{c}\text { Maximum out-of-plane } \\
\text { displacement }\end{array}$ & $0.4 \mathrm{~mm} / 0.02 \mathrm{~mm}$ \\
\hline Peak blocked force & $0.284 \mathrm{~kg} / 0.04 \mathrm{~kg}$ \\
\hline $\begin{array}{c}\text { Blocked force at room } \\
\text { temperature }\end{array}$ & $0.23 \mathrm{~kg} / 0.02 \mathrm{~kg}$ \\
\hline $\begin{array}{c}\text { Activation temperature } \\
\text { force }\end{array}$ & $\sim 40^{\circ} \mathrm{C}$ \\
\hline $\begin{array}{c}\text { Temperature at peak blocked } \\
\text { Temperature to reconfigure }\end{array}$ & $\sim 38^{\circ} \mathrm{C}$ \\
\hline $\begin{array}{c}\text { Rate of cooling at room } \\
\text { temperature }\left(26^{\circ} \mathrm{C}\right)\end{array}$ & $\sim 3.61^{\circ} \mathrm{C} / \mathrm{s}$ \\
\hline
\end{tabular}

\section{ERAsable Tactile Drawing Interface}

For students with visual impairments, drawing can be of equal importance as it is for their sighted peers in scientific thinking, learning and creativity [15]. At present, there are few tactile interfaces with which students with visual impairments can draw and erase to learn and express themselves. Therefore, in this section, we will describe the design and results from an initial user evaluation of a manual interface that uses Tacilia to enable reading, drawing, and erasing tactile diagrams.

\section{A. Design of a Hot Air Pencil}

Tactile pixels on Tacilia can be selectively actuated with an external source of heat. If this source is in the form of a pencil, it can enable a manual tactile drawing interaction with Tacilia. In our concept, the pencil emits a jet of hot air to actuate the pixels. When this jet touches the sheet, it will raise the temperature of a localized region and actuate all the pixels in that region. The speed and accuracy of drawing depends on the spread of the jet, which is depended on the diameter of the nozzle, the air pressure at exit, distance of the nozzle from the sheet and the temperature of the exiting air.

The mechanical design of the pencil is inspired from a double barrel vacuum flask which insulates the heat to actuate the pixels from the fingers holding the pencil. The barrel of the pencil is made from Bakelite to reduce heat transfer through conduction. Vacuum is created between the two barrels to further reduce heat transfer through convection. Air is pumped through the inner barrel of the pencil, which contains a heater. The heater is made from

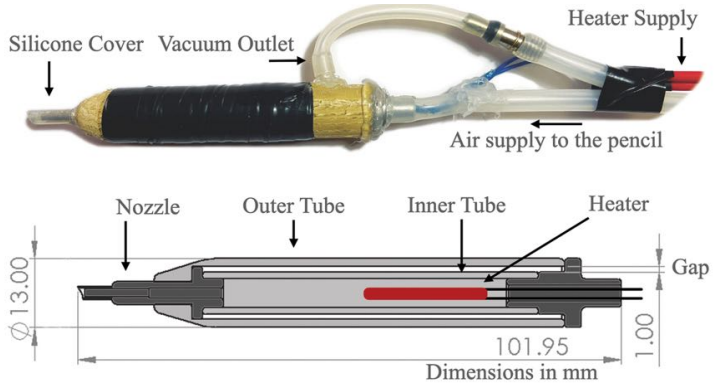

Figure 6. Prototype and schematic diagram of the pencil.

Nichrome wire and consumed $15 \mathrm{~W}$ while the DC air pump is rated for a maximum of $6 \mathrm{~V}, 0.15 \mathrm{~A}$. The incoming air from the pump gets heated and exits from a nozzle at the tip of the pencil. The diameter of the nozzle is $0.2 \mathrm{~mm}$. An extended silicone tube covers the nozzle that maintains a gap of $0.5 \mathrm{~mm}$ between the nozzle and the sheet to provide room for the pixels to actuate. It disallows direct contact of the fingertips with the exiting air and the heated pixels. It also concentrates the air exiting from the nozzle over the target area. At $6 \mathrm{~V}$, the air pump generates $380 \mathrm{mmHg}$ pressure at the exit of the silicone cover. In 30 seconds, the temperature of the heater reaches $270^{\circ} \mathrm{C}$ from ambient temperature. At this temperature and air pressure, the temperature of hot air exiting the nozzle reaches around $120^{\circ} \mathrm{C}$ which instantaneously actuates the pixels. The average time for targeted actuation (from 5 randomly selected pixels) is $0.6 \mathrm{~s}$ whereas for freehand drawing, it takes an average of $1 \mathrm{~s}$ for a pixel to actuate.

\section{B. User Evaluation of the Interaction with Blindfolded Participants}

We were interested to study two usability aspects: readability on Tacilia and freehand creation of tactile drawings. We systematically evaluated the two aspects individually with 5 blindfolded, sighted participants, age between 24 - 59 years (mean 40 years), out of which 3 were males and 2 were females. Each participant was neurologically healthy and reported no problems with the sensory integrity of their fingertips. They also had no Braille or tactile graphic literacy. In a one-on-one session, the participant was seated on a study table in a comfortable, well ventilated room and the display device was presented to them. After the participants were informed about the study and consented to participate, they were blindfolded, and the session was conducted in a sequence of three studies.

\section{1) Study 1: Absolute Threshold of Scale}

The aim of the first study was to identify the threshold of the smallest square which was perceivable on the tactile pixel array. We followed the psychophysics method of constant stimuli to determine this threshold. Three columns of squares were drawn in an ascending order of scale from a $2 \times 2$ to $6 \times 6$ pixel array. In the first round, filled squares were presented while in the second round only outlines of these squares were presented (Figure 7a). Participants were asked to explore the shapes from bottom to top on the first and the third column and top to bottom for the second column. This was done to avoid habituation. While exploring the squares, we asked them for a forced choice on whether the shape was clearly perceivable as a square or not clearly perceivable. The scale 
at which participants were able to clearly perceive the square more than $50 \%$ of the time on average was recorded.

Participants only used their dominant hand's index finger to read the tactile diagrams. They expressed a greater confidence in recognizing squares through their outlines made with a single line of actuated pixels in comparison to completely filled squares. The tactile contrast between the rough actuated pixels with respect to their flat surroundings on both the sides enabled a clearer perception of the shape. A $3 \times 3$ pixel outlined square was perceived clearly as a square $73 \%$ times by the participants. In comparison, only a $4 \times 4$ solid filled square was perceived clearly $53 \%$ of the time. A $2 \times 2$ squares were not perceived as a square by any participant whereas $5 \times 5$ outlined squares were instantly recognized by all of the participants. This shows that larger tactile shapes are more perceivable than smaller tactile shapes on the display. This is concurrent with existing findings from tactile shape perception with raised line drawings [16].
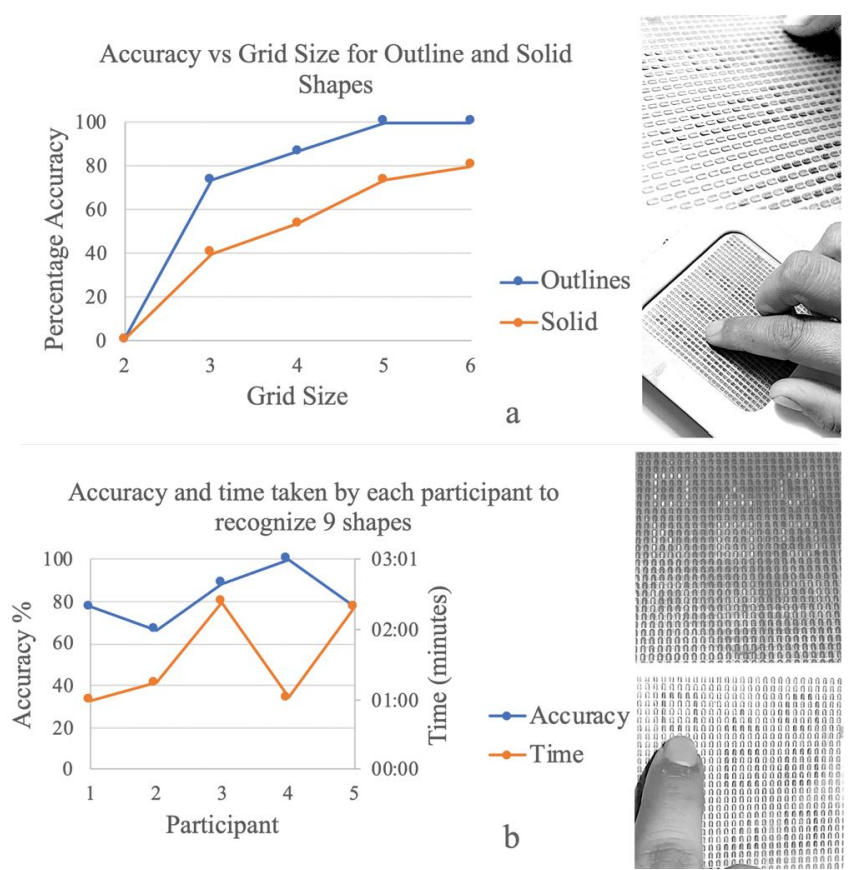

Figure 7. (a) Plot between perceptual accuracy and grid size of squares. (b) Accuracy of perception of shapes and time taken by each participant.

\section{2) Study 2: Discrimination of Shapes}

The aim of the second study was to evaluate if participants are able to clearly differentiate between shapes on the tactile display. Participants read 9 pre-drawn outlined shapes made in 3 rows and 3 columns. These were a square, triangles (isosceles and right angled), and circle. Every row and column had one of the three shapes. All the shapes were made in a $5 \times 5$ pixel array (Figure $7 \mathrm{~b}$ ). It is important to note that the circle was made by erasing the pixels of the vertices of the square.

Participants were asked to read the shapes column-wise. They only used the index finger of their dominant hand to read the shapes. Overall, the accuracy of correctly detecting the 9 shapes was $82.2 \%$, which took an average of $96 \mathrm{~s}$ to complete. The average accuracy for shape detection in the first column was $66.6 \%$. In the second column the accuracy rose to $93.3 \%$ and it was $86.6 \%$ for the third column. This shows that the accuracy of perception can increase with familiarity. The accuracy for detecting tringles was $80 \%$, for squares it was $86.6 \%$ and for circles it was $80 \%$. Despite the minor difference between the square and the circle, participants were able to differentiate between the two shapes $83.3 \%$ of the time. This shows that even a single pixel difference is perceivable on the display to a novice reader.

\section{3) Study 3: Creating and Erasing Tactile Drawings}

In the third study, we made qualitative observations of the initial interaction to create and erase tactile drawings with the hot-air-jet pencil. The goal of the study was to observe how participants instinctively learn to draw freehand shapes only through the tactile sensations.

After familiarizing themselves with the interface for 10 minutes, we asked blindfolded participants to draw two alphabets of the English language of their choice, thrice on the display and erase them after each attempt. Their subjective feedback along with the outcome and the time it took them to draw was recorded (Figure 8).

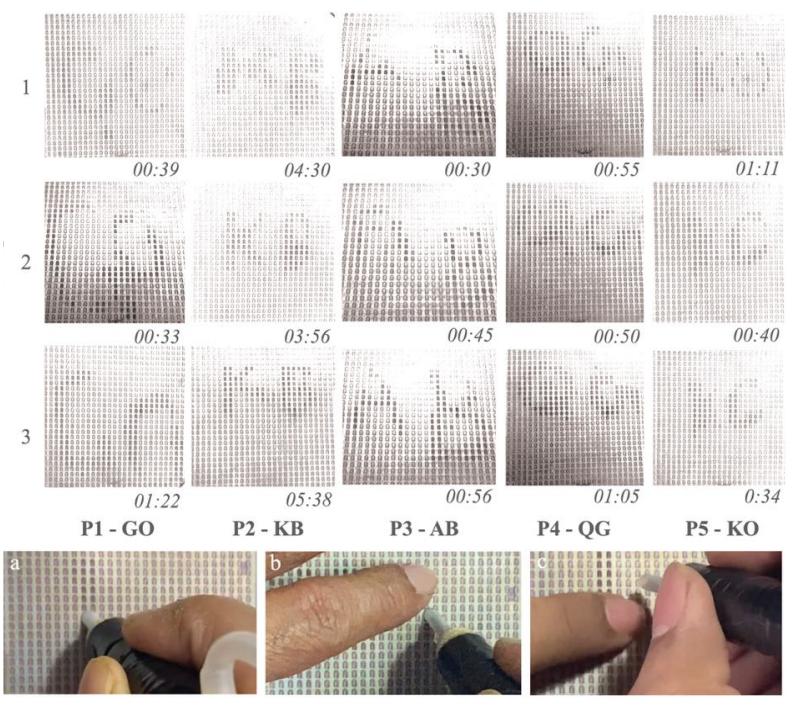

Figure 8. Successive attempts on drawing alphabets by the participants with the time they took to draw (in minutes) (a) Drawing freehand with tactile feedback from the pencil (b) drawing with consistent feedback from the fingertips of the other hand (c) erasing tactile pixels.

In general, drawing vertical and horizontal lines was considered to be easier. Drawing curves and diagonal lines was difficult and had to be improvised through self-learning. It was easier to create large shapes with fewer details and drawing finer details was regraded to be difficult and time consuming. Erasing was regarded as very easy as participants intuitively used their fingertips to flatten selected pixels.

Tactile drawing was slower compared to pen-paper based sighted drawing (ranging from $30 \mathrm{~s}$ to $338 \mathrm{~s}$ for two alphabets). However, the speed of drawing varied across participants and was depended on their autodidactic style of drawing. P1, P3 and P5 drew predominantly through the feedback from the pencil. The compliant silicone deformed when it encountered an actuated pixel, which was felt by the hand holding the pencil as a low frequency vibration. Fingertips of the other hand were therefore used only for overall shape perception after drawing or during intermittent pauses to check what has been drawn. With this compliant 
extension, users were also able to selectively actuate targeted pixels. P5 reflected that practice-based learning was happening and that with each attempt they were becoming more comfortable and confident about how to draw tactile shapes. It took them an average of $47.7 \mathrm{~s}$ to draw.

Differently from the above, P4 glided over the pixels without touching them while following the trajectory of the intended curve through constant feedback from the fingertips of the other hand. This was a fast and accurate strategy which took $55 \mathrm{~s}$ on average and produced satisfactory results for the participant.

P2 on the other hand, created accurate characters by coordinating actions of actuating, perceiving and erasing pixels. Although this strategy provided a greater control and accuracy of the drawing, it took P2 more time to draw in comparison to other participants ( $\sim 4 \mathrm{~min}$ on average). P2 also developed a unique way to create diagonal lines by following the intended diagonal trajectory slower than the straight-line movements. This actuated all the pixels in the path, some of them pixels which were intermittently erased to increase the accuracy of the shape.

\section{DiscuSSION AND FUTURE WORK}

We have demonstrated a method that transforms a single monolithic sheet of Nitinol into a passive shape-changing tactile pixel array at Braille resolution. Preliminary design and engineering details of this passive shape changing interface are discussed, which takes a previously suggested concept for tactile display actuators forward [6]. We demonstrate Tacilia's potential to be used as an erasable tactile drawing canvas, for which we have also discussed the design of a specialized pencil and presented a preliminary observation of the interaction with blindfolded novice tactile readers.

In the current prototype, vertical displacement of the tactile pixels is less than the height of a standard Braille dot. The design of the pixels can be optimized to achieve a greater vertical displacement through an overlapping $\mathrm{V}$-shaped pixel arrangement. It will increase the length of the bending cantilevers which reduces the strains formed at the base of the pixels, increasing their propensity to bend higher without compromising on the strength of the overall sheet. In future works, we will investigate this design concept and also study the fatigue life of the thermo-mechanical actuation.

A faster actuation of the pixels can lead to a more intuitive drawing interaction with greater accuracy. Faster actuation can be achieved either by reducing the activation temperature of the sheet's SMA material or by increasing the temperature of the hot-air-jet. Even with the present heatbased interaction, participants were able to adapt with the hot-air-jet for their safety and no heat related injuries were reported. However, the former solution will enable interaction at a lower temperature that is both power efficient and safer for manual interaction.

To achieve a programmable actuation of the pixels, we will have to design and fabricate an array of heaters which can be selectively heated. These have to be mechanically aligned to the shape changing pixels. An array of pins on top of Tacilia can therefore be selectively raised to present
Braille and tactile diagrams. Reconfigurability will require a mechanical mechanism to press the actuated pixels back to their flat state. In future work, we intend to integrate the heating and reconfiguration subsystems to develop an actuator suitable for digital refreshable Braille and tactile graphic displays.

\section{ACKNOWLEDGMENT}

We thank our participants, Vikas Upadhyay and technicians of PE Lab at IIT Delhi who helped in the fabrication of the prototype. We also thank our reviewers for their feedback on the manuscript.

\section{REFERENCES}

[1] V. G. Chouvardas, A. N. Miliou, and M. K. Hatalis, "Tactile displays: Overview and recent advances," Displays, vol. 29, no. 3, pp. 185-194, Jul. 2008, doi: 10.1016/j.displa.2007.07.003.

[2] S. W. Robertson, A. R. Pelton, and R. O. Ritchie, "Mechanical fatigue and fracture of Nitinol," International Materials Reviews, vol. 57, no. 1, pp. 1-37, Jan. 2012, doi: 10.1179/1743280411Y.0000000009.

[3] P. Sapra et al., "A Compliant Mechanism Design for Refreshable Braille Display Using Shape Memory Alloy," presented at the ASME 2015 International Design Engineering Technical Conferences and Computers and Information in Engineering Conference, Jan. 2016. doi: 10.1115/DETC2015-47468.

[4] R. Velázquez, E. Pissaloux, and M. Wiertlewski, "A compact tactile display for the blind with shape memory alloys," Jun. 2006, vol. 2006, pp. 3905-3910. doi: 10.1109/ROBOT.2006.1642300.

[5] J. Xu et al., "Fabrication and characterization of SMA film actuator array with bias spring for high-power MEMS tactile display," Microelectronic Engineering, vol. 227, p. 111307, Apr. 2020, doi: 10.1016/j.mee.2020.111307.

[6] M. Hafez and J.-M. Alexandre, "Touch-sensitive interface," US20060154216A1, Jul. 13, 2006 Accessed: Feb. 10, 2021. [Online]. Available: https://patents.google.com/patent/US20060154216A1/en

[7] "(PDF) Experimental study on two way shape memory effect training procedure for NiTiNOL shape memory alloy," ResearchGate. https://www.researchgate.net/publication/283807098_Experimental_st udy_on_two_way_shape_memory_effect_training_procedure_for_ $\overline{\mathrm{N}} \mathrm{i}$ TiNOL_shape_memory_alloy (accessed Apr. 18, 2020).

[8] "Standards," $U K A A \bar{F}$. https://www.ukaaf.org/braille/standards/ (accessed Apr. 18, 2020).

[9] M. A. Darden and C. J. Schwartz, "Skin tribology phenomena associated with reading braille print: The influence of cell patterns and skin behavior on coefficient of friction," Wear, vol. 332-333, pp. 734-741, May 2015, doi: 10.1016/j.wear.2014.12.053.

[10] "Introduction to Nitinol," p. 41, 2017.

[11] "Nitinol - NiTi Shape Memory Alloy; High-Temperature Phase." http://www.matweb.com/search/datasheet.aspx?MatGUID=de9dd084 33714f698d513766dccea437 (accessed Apr. 23, 2020).

[12] "SMA Shape Training Tutorial," p. 46.

[13] M. Nishida and T. Honma, "All-round shape memory effect in Ni-rich TiNi alloys generated by constrained aging," Scripta Metallurgica, vol. 18 , no. 11 , pp. 1293-1298, Nov. 1984, doi: 10.1016/00369748(84)90125-X.

[14] E. Ungar, "A New Approach to Defining Human Touch Temperature Standards," presented at the 40th International Conference on Environmental Systems, Barcelona, Spain, Jan. 2010. Accessed: May 05, 2020. [Online]. Available: https://ntrs.nasa.gov/search.jsp?R=20100020960

[15] S. O’Modhrain, N. Giudice, J. Gardner, and G. Legge, "Designing Media for Visually-Impaired Users of Refreshable Touch Displays: Possibilities and Pitfalls," IEEE transactions on haptics, vol. 8, Aug. 2015, doi: 10.1109/TOH.2015.2466231.

[16] M. W. A. Wijntjes, T. van Lienen, I. M. Verstijnen, and A. M. L. Kappers, "The Influence of Picture Size on Recognition and Exploratory Behaviour in Raised-Line Drawings," Perception, vol. 37, no. 4, pp. 602-614, Apr. 2008, doi: 10.1068/p5714. 